\title{
Application of PTP synchronized PMUs in power system small-signal stability analysis
}

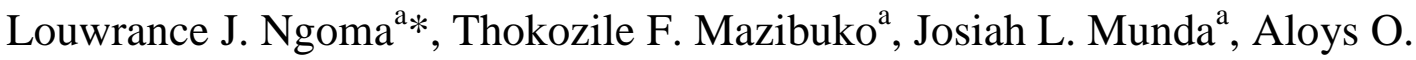 \\ Akumu $^{\mathrm{a}}$
}

${ }^{a}$ Tshwane University of Technology, Department of Electrical Engineering, Pretoria, 0001, South Africa

\begin{abstract}
Small-signal stability problem is one of the major threats to the power system network security and reliability. Accurate and timely information about the oscillation modes can help optimize stability margin settings, as well as give early warnings for unstable modes to operate a power system at its full capacity while staying within the stability boundaries. This paper considers the possibility of using a software-only Precision Time Protocol (PTP) to synchronize a number of PMUs and how these synchronization uncertainties impact the monitoring of low-frequency oscillations. In the proposed scheme Prony analysis is realized to estimate signals damping information. The proposed scheme is applied on a two-area power system. Simulation results point to the success of the proposed scheme in monitoring of low-frequency oscillations.
\end{abstract}

Keywords: Phasor Measurement Unit, low-frequency oscillations, Precision Time Protocol, State Estimation, Prony analysis

\section{Introduction}

Synchronized Phasor Measurement Units (PMUs) applications have been considered in several literatures, and no doubt many more applications will be established in coming years [1]. One of the utmost important applications of PMUs in Wide Area Monitoring Systems (WAMS) is monitoring of low-frequency oscillations. Synchronization between PMUs is accomplished by same-time sampling of the voltage and the current waveforms using a common synchronizing signal from the Global Positioning System (GPS) [2]. However, different synchronization solutions exist but differing from each other in terms of functional features, complexity, performance and costs. An open source software implementation for Linux environment of the PTP, named Precision Time Protocol Daemon (PTPd), has been used to synchronize general computers [3].

The results of performance evaluation of IEEE 1588 Clock Synchronization technology realized on the x86 (Intel) Linux platform were presented in [4]. A Software-only PTP IEEE 1558 was implemented using general computers with minimal hardware to study the prospect of using a software-only PTP (Precision Time Protocol) synchronization technique to synchronize various PMUs inside the same substation and how this synchronization affects the State Estimation [5]. The application of synchronized PMUs only requires that the power system should be completely observable through phasor measurement units only for power system state estimation application. According to $[1,5,6]$, it is possible to find several algorithms for state estimation: some completely based on traditional measures, some of which make a combined use of traditional measures and synchrophasors and others completely based on PMU measurements. A power system with a sufficient number of installed PMUs at appropriate locations for a fully observable system is considered in [5]. With the fully observed system, the state estimation problem

\footnotetext{
* Manuscript received August 5, 2013; revised November 4, 2013.

Corresponding author. Tel.: +27 76926 6584; E-mail address: ngomalj@ gmail.com.
} 
becomes a linear problem solvable using only data provided by PMUs and many issues associated with traditional state estimators could be resolved.

This paper seeks to implement the PTP IEEE 1588 synchronization protocol for synchronizing two PMUs, because of its advantages of low cost and less complexity, and to experimentally evaluate the synchronization performances of the PTP (software time stamping mechanism) protocol and the effect of its accuracy in power system small-signal stability applications. Prony analysis is used to monitor power system small signal stability by estimating signal damping information.

\section{Synchronized Phasor Measurement Units}

\subsection{PTP System}

A PTP system is a distributed networked system, which includes devices such as peer-to-peer transparent clocks, boundary clocks, ordinary clocks, end-to-end transparent clocks, and management nodes devices. It also includes non-PTP devices i.e. routers, bridges. The clocks in a distributed system are structured into a master to slave synchronization network where the top clock in the network defines the reference clock for the whole system. This clock is known as the grand master clock. PTP timing messages are exchanged between the clocks to achieve synchronization. The slaves use the timing data to regulate their clocks to the master clock in the network [7].

\subsection{Phasor definition}

Phasor representation of sinusoidal waveforms is usually used in ac power system analysis. Consider a sinusoidal signal defined in (1):

$$
x(t)=X_{m} \cos (\omega t+\emptyset)
$$

where the signal magnitude is $X_{m}, \omega=2 \pi f$ is the system pulsation, $\emptyset$ is the initial phase angle which depends on the time scale, mostly where $t=0$. The phasor representation of this sinusoidal signal is given by (2):

$$
X=\frac{X_{m}}{\sqrt{2}} e^{j \emptyset}=\frac{X_{m}}{\sqrt{2}}(\cos (\varnothing)+j \sin (\varnothing))=X_{r}+X_{i}
$$

where the rms value of the signal magnitude is $\frac{X_{m}}{\sqrt{2}}, X_{r}$ and $X_{i}$ indicate, respectively, the real and imaginary parts of a complex phasor representation in rectangular components [8].

The synchrophasor representation of the signal $x(t)$ in (1) is the value $X$ in (2) where $\emptyset$ is the instantaneous phase angle relative to a cosine function at the nominal system frequency synchronized to UTC.

\subsection{Measurement performance}

A synchrophasor measurement is evaluated using Total Vector Error (TVE). The standard defines TVE as the difference between a perfectly synchronized theoretical synchrophasor and the estimated phasor given by the unit under test at the same instant of time. To put it more simply, TVE is the relative difference between a measured phasor at the output of the PMU and a reference phasor [8]:

$$
T V E=\sqrt{\frac{\left(\hat{A}_{r}(n)-A_{r}(n)\right)^{2}+\left(\hat{A}_{i}(n)-A_{i}(n)\right)^{2}}{A_{r}(n)^{2}+A_{i}(n)^{2}}}
$$

where: $A_{r}(n)$ and $A_{i}(n)$ are sequences of theoretical real and imaginary parts of the input signal at the instants of time $(n)$ assigned by the unit to those values and $\hat{A}_{r}(n)$ and $\hat{A}_{i}(n)$ are sequences of real and 
imaginary parts of the measured synchrophasor quantities at an instant of time $(n)$ by the PMU under test.

TVE represents a mixture of two potential errors, i.e. the magnitude error and the phase error. For instance, if a given PMU that is perfectly synchronized with the reference time has no phase error, $1 \%$ of TVE means that this total vector error is caused by $1 \%$ of the magnitude error. Similarly, with a zero magnitude error, $1 \%$ of the TVE is due to $1 \%$ of the phase error which is 0.01 radians or 0.570 degrees. This corresponds to a time error of approximately $\pm 31 \mu \mathrm{s}$ for a $50 \mathrm{~Hz}$ system [8].

\section{State Estimation}

In this paper, a Weighted Least Squares (WLS) estimator, which is realized using only voltage and current measurements, is considered. The problem for any state estimation method is to solve for the system states (bus voltages and angles) based on obtainable data. The state estimation problem is shown by (4):

$$
[z]=\left[\begin{array}{c}
\Pi \\
{[y][A]+\left[y_{s}\right]}
\end{array}\right]\left[E_{B}\right]
$$

where,

$z: \quad$ Set of voltage and current phasor measurements

$\Pi$ : $\quad$ Voltage measurement bus incidence matrix

$y$ : $\quad$ Series admittance matrix

$y_{s}:$ Shunt admittance matrix

$A$ : Current measurement bus incidence matrix

$E_{B}$ : $\quad$ System state variables (all bus voltages)

and,

$$
\begin{aligned}
& B=\left[\begin{array}{c}
\Pi \\
{[y][A]+\left[y_{s}\right]}
\end{array}\right] \\
& {[E]=\left[\left(B^{T} W^{-1} B\right)^{-1} B^{T} W^{-1}\right][z]}
\end{aligned}
$$

where $W$ is Measurement covariance matrix.

The state estimation formulation proposed can be extended using the technique described in [1].

\section{Prony Analysis}

Prony analysis has been presented to be a feasible method to model a linear sum of damped complex exponentials to signals that are uniformly sampled. Prony analysis is a system identification technique, which is commonly used in the power system electromechanical oscillation estimation application [9].

The Prony analysis directly estimates the parameters by fitting a sum of complex damped sinusoids to evenly spaced sample (in time) values of the output:

$$
\hat{y}(t)=\sum_{i=1}^{n} A_{i} e^{\left(\sigma_{i} t\right)} \cos \left(2 \pi f_{i} t+\emptyset_{i}\right)
$$

The samples of $\hat{y}(t)$ can be written as:

$$
\hat{y}(k T)=\sum_{i=1}^{n} B_{i} z_{i}^{k}
$$

where $B_{i}$ is an output residual for $\lambda_{i}$ eigenvalue. 


$$
B_{i}=\frac{A_{i}}{2} e^{j \emptyset_{i}}
$$

$z_{i}$ is the discrete pole of the system and $t=k T$

$$
z_{i}=e^{\left(\sigma_{i}+j 2 \pi f_{i}\right) T}
$$

and $T$ is the sampling period.

The damping $\alpha_{i}$ and sinusoidal frequency $f_{i}$ may be determined from the root $z_{i}$ using the relationships:

$$
\begin{aligned}
\alpha_{i} & =\frac{\ln \left|z_{i}\right|}{T} \\
f_{i} & =\frac{\tan ^{-1}\left[\frac{\lim \left(z_{i}\right)}{\operatorname{Re}\left(z_{i}\right)}\right]}{2 \pi T}
\end{aligned}
$$

The amplitude $A_{i}$ and the phase $\theta_{i}$ may be determined from each $B_{i}$ parameter with the relationships:

$$
\begin{aligned}
& A_{i}=\left|B_{i}\right| \\
& \theta_{i}=\tan ^{-1}\left[\frac{\operatorname{im}\left(B_{i}\right)}{\operatorname{Re}\left(B_{i}\right)}\right]
\end{aligned}
$$

\section{Characteristics of Small-Signal Stability Problems}

In large power systems, small-signal stability problems may be either local or global in nature. Power system disturbances, such as variation of generation, cause local and inter-area oscillations. Usually, local oscillation modes range in frequency from 0.8 to $2.0 \mathrm{~Hz}$. Inter-area oscillation, which refers usually to a group of generators in one area that swing against a group of generators in another area, normally ranges in frequency from 0.2 to $0.8 \mathrm{~Hz}$ [10].

The stability in the small signal of a nonlinear system is given by the roots of the characteristic equation of the system and is given by Lyapunov's first method [10]. When $\sigma_{i}<0$ the system is asymptotically stable, $\sigma_{i}>0$ the system is unstable and when $\sigma_{i}=0$ it is not possible to say anything in general.

\section{Tests on the PTP Synchronization System}

The PTPd implementation has been tested in a test environment shown in Fig. 1. Both the PTP master and slaves are implemented using PTPd v2 (software time stamping) running on current PC hardware.

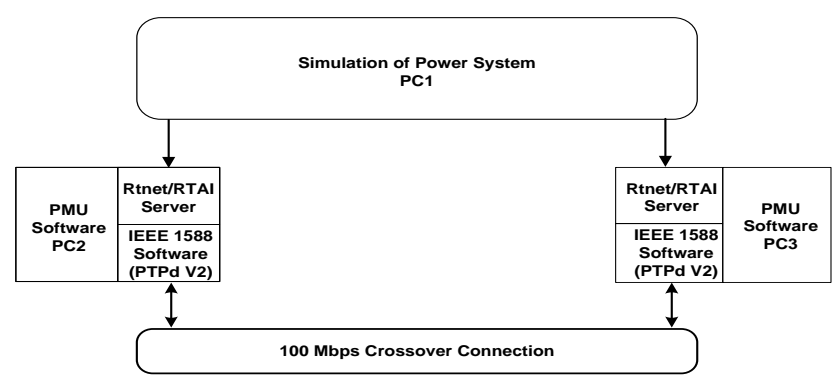

Fig. 1. PTP/PMUs test setup. 
The following plots report the accuracy performance of the software-only synchronization system. Fig. 2 shows the synchronization offset trend between the master and slave clocks for the whole sixty minutes duration test. Fig. 3 plots the next 3120 samples (52 minutes) after the initial convergence period. The offset remains below $4.5 \mu \mathrm{s}$ after ten minutes until the end of the test, thus the PTPd implementation achieves sub-microsecond precision. The accuracy of synchronization appears to be compatible with the requirements of [8]

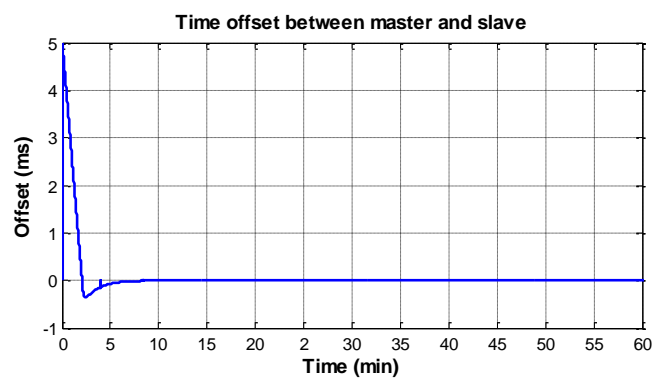

Fig. 2. Synchronization offset, 0-60 minutes

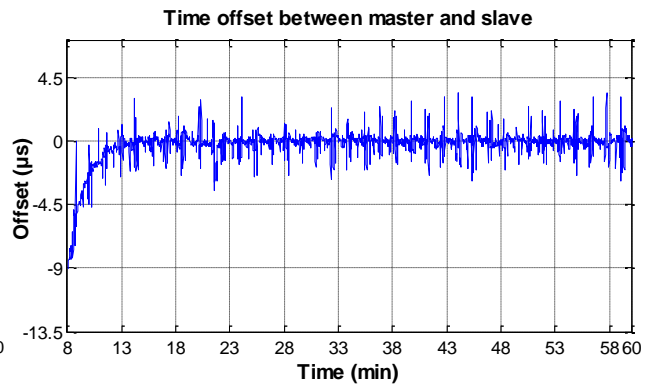

Fig. 3. Synchronization offset, 8-60 minutes

In Fig. 4 the frequency distribution of the synchronization offset in minutes 10 to 60 is reported. The histogram contains $0.1 \mu \mathrm{s}$ bins with 3,000 offset samples (fifty minutes). It can be observed that the offset distribution is within $\pm 4.5 \mu \mathrm{s}$ and is concentrated at the region within $\pm 1.5 \mu \mathrm{s}$. In Table 1 the standard deviation and average value of the synchronization offset are reported. The implementation achieves accuracies of about $\pm 4.5 \mu \mathrm{s}$ as a pure software solution.

One significant concern arising from the application of a completely software assisted PMU concerns the latency of the operating system. In Fig. 5 the distribution of the overall jitter that affects the timestamping process of every sample is reported and, in Table 2 values of mean and standard deviation are reported. It is important to notice that, different from the synchronization offset that is characterized by a mean value almost equal to nanoseconds, the mean value of the complete time jitter is in the order of 2.8 microseconds. However, as far as the requirements enforced by [8] are concerned, the TVE 1\% limit would be reached if only the time synchronization error is equal to $31.8 \mu \mathrm{s}$ at $50 \mathrm{~Hz}$. Thus from the synchronization results, the synchronization offset values are way below the minimum value that can contribute an error causing the TVE to be near the $1 \%$ limit.

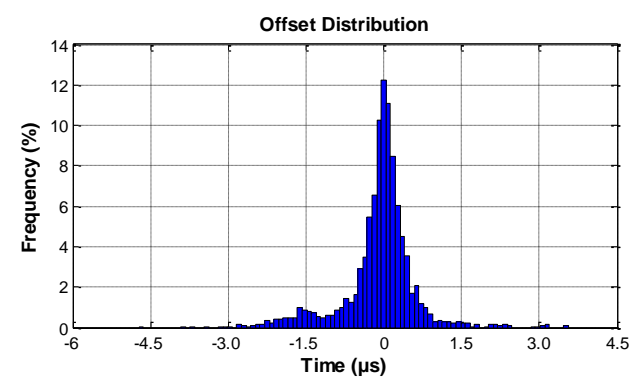

Fig. 4. Synchronization offset distribution

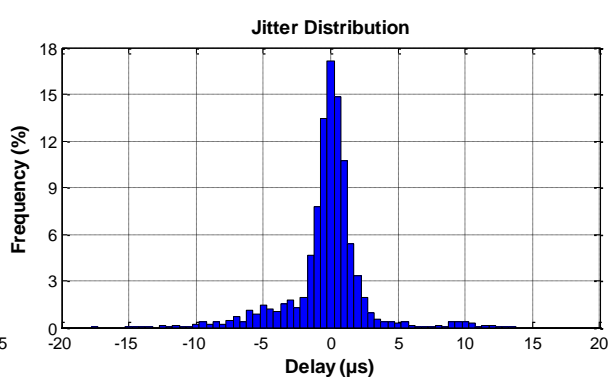

Fig. 5. Jitter distribution of sample time-stamps

Table 1. Synchronization offset standard deviation and average value

\begin{tabular}{ccl}
\hline Standard Deviation $(\boldsymbol{\mu s})$ & Average value $(\boldsymbol{\mu s})$ & No. of Samples \\
\hline 0.41257 & -0.091389 & 3000 \\
\hline
\end{tabular}

Table 2. Standard deviation and average of jitter of sample time stamps

\begin{tabular}{ccc}
\hline Standard Deviation $(\boldsymbol{\mu} \mathbf{s})$ & Average value $(\boldsymbol{\mu} \mathbf{s})$ & No. of Samples \\
\hline 0.24853 & 2.8245 & 3000 \\
\hline
\end{tabular}




\section{Tests on the Synchrophasor Measurement System}

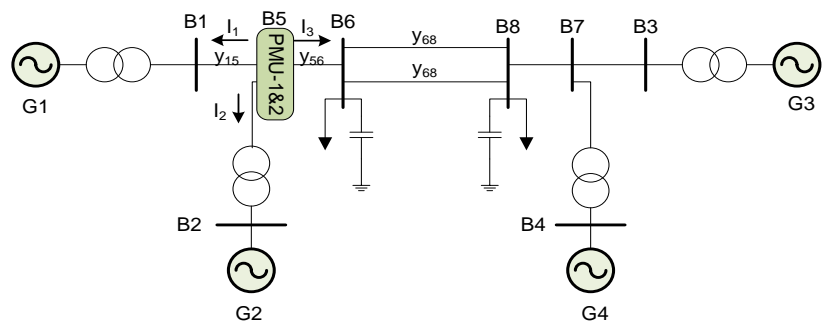

Fig. 6. Power system model

PMU errors are calculated using the realized TVE function. This function receives the estimated phasors of PMU-1 and PMU-2 as shown in Fig. 6, compares the two sets of parameters in every time frame and estimates the differences. Apart from TVE, the function also determines PMU magnitude error and phase error.

In Fig. 7 and 8, the TVE and phase error distributions are reported. These results show that not only the TVE for the current signal never reach values bigger than $0.4 \%$, but also that in the greater part of the cases the TVE assume values much smaller than $0.4 \%$. It can be observed that the phase difference never reaches a value greater than $3.7 \mathrm{mrad}$. Thus, from these results it can be safely concluded that the realized PMUs and synchronization accuracy meet the requirements in [8] .

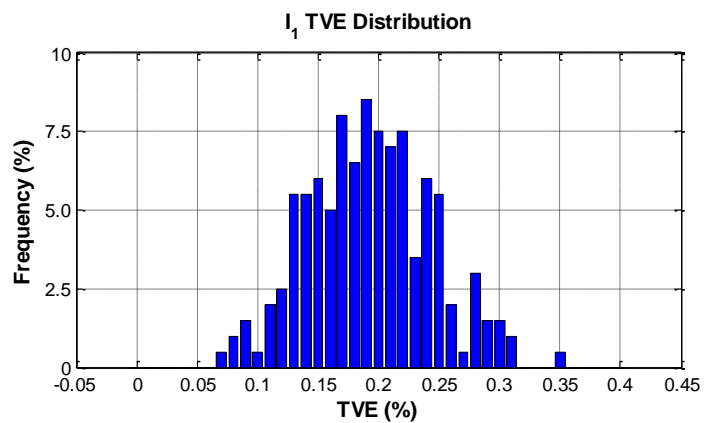

Fig. 7. $\mathrm{I}_{1}$ TVE distribution

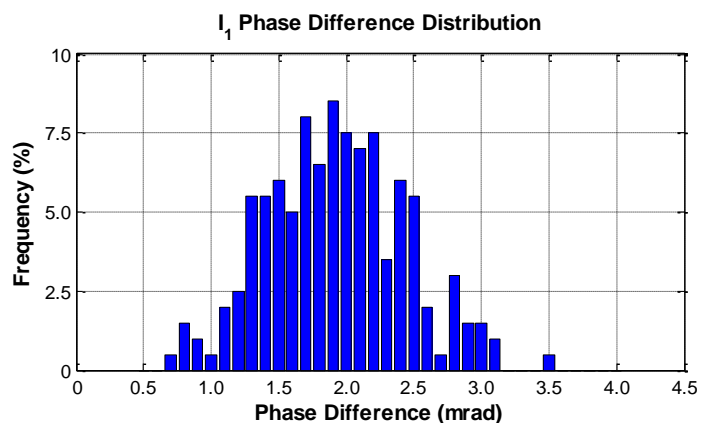

Fig. 8. $\mathrm{I}_{1}$ phase error distribution

Table 3: Synchrophasor TVE and phase error when the two PMUs acquire the same signal

\begin{tabular}{cccc}
\hline Signal & Average TVE $(\%)$ & Average phase error $(\mathrm{mrad})$ & No of Samples \\
\hline$I_{1}$ & 0.1925 & 1.9 & 1500 \\
$I_{2}$ & 0.1889 & 1.4 & 1500 \\
$I_{3}$ & 0.0428 & 1.9263 & 1500 \\
$V_{5}$ & 0.1875 & 1.9 & 1500 \\
\hline
\end{tabular}

\section{Tests on the State Estimation Application}

To implement state estimation of the considered power system in Fig. 9 by using only synchrophasor data, the PMUs have to be properly located. The problem of PMUs placement has been achieved in Fig. 9 to place the minimum number of PMUs for complete observability in case of normal operation. The state estimation using the Weight Least Square method is realized on Matlab environment and performed offline. The two virtual PMUs acquire all the signals (voltages and currents) of the equivalent bus. A reference case has been created: the digital time domain values of the measured signals for bus 5 and 7 are exported from the simulation using Real Time Workshop (RTW) standard devices to a text file and are used to calculate the phasors. The reference case considers all synchronization and acquisition errors equal to zero. Two cases for state estimation were considered, the first case is that the output data from 
the reference PMUs (perfectly synchronized) is used to estimate the state of the simulated power network. Second case, the data of the synchronized PMUs is used to estimate the state of the power network. Figures 10-12 show $V_{5}$ state variable of the reference case compared with the synchronized PMUs case in terms of TVE and phase error.

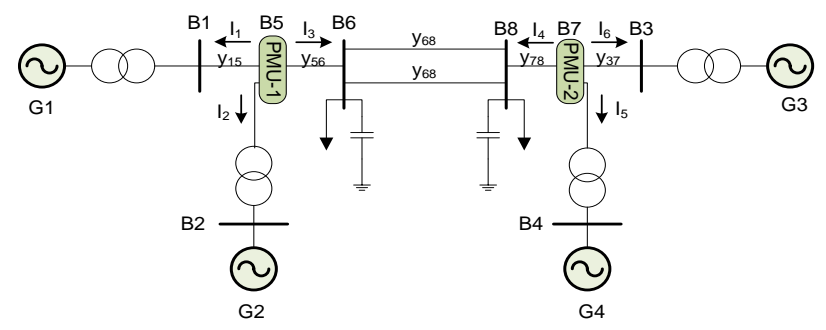

Fig. 9. Power system model showing current measurements

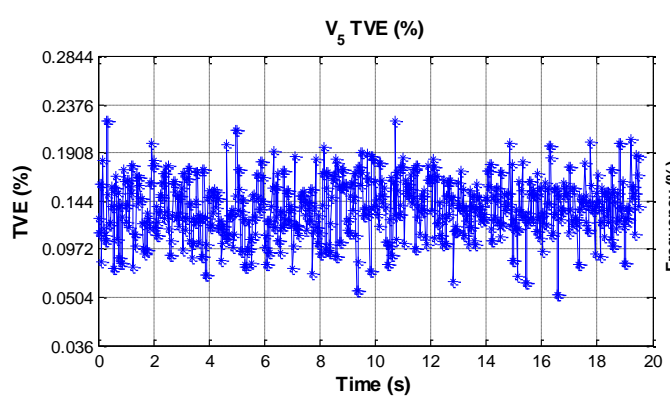

Fig. 10. V5 TVE trend

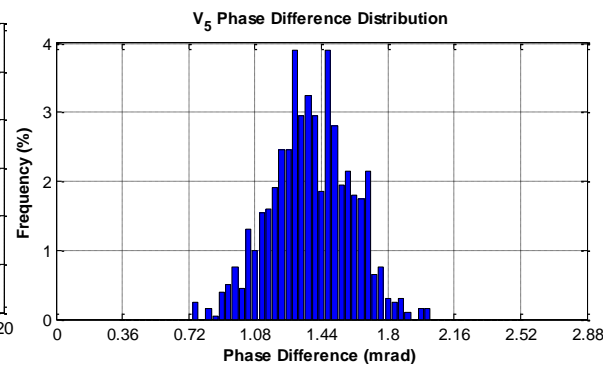

Fig. 11. $V_{5}$ phase difference distribution

The average and standard deviation of the system state variables TVE and phase error are reported in Table 4. It can be seen that with the application of the state estimation the TVE and phase error have decreased compared to Table 3 , the average values are within the expected value of $\pm 1.3 \mathrm{mrad}$, thus it can be concluded that the application of state estimation Weight Least Square with the inclusion of the measurement error covariance matrix which serves to weigh the accuracy of each of the measurements, the effect of the synchronization error is reduced.

Table 4. Synchrophasor TVE and phase error for State estimation

\begin{tabular}{ccc}
\hline Signal & Average TVE $(\%)$ & Average phase error (mrad) \\
\hline$V_{1}$ & 0.1357 & 1.3493 \\
$V_{2}$ & 0.1382 & 1.3644 \\
$V_{3}$ & 0.1375 & 1.3345 \\
$V_{4}$ & 0.1415 & 1.3385 \\
$V_{5}$ & 0.1390 & 1.3838 \\
$V_{6}$ & 0.1357 & 1.3439 \\
$V_{7}$ & 0.1361 & 1.3522 \\
$V_{8}$ & 0.1354 & 1.3486 \\
\hline
\end{tabular}

The fact that the electromechanical oscillations are relatively slow (in the $0.2-2.0 \mathrm{~Hz}$ range) is an encouraging factor for synchronizing PMUs with PTP software time stamping mechanism, since the synchronization error brought by the uncertainty of the PTP software only (in the range of $\pm 4.5 \mu \mathrm{s}$ ) would bring a vector error of $0.0065 \%$ for a $2 \mathrm{~Hz}$ oscillation and that has a minimal impact on the electromechanical oscillation monitoring application. 


\section{Prony Analysis Simulation Results}

Prony analysis of the PMUs data is realized offline on Matlab environment to analyse the system tie line 6-8 signal parameters and determine the different modes of the system. The power system local and inter-area oscillations are achieved by small disturbance for the generator 1 reference voltage step from $1.0 \mathrm{p} . u$ to $1.05 \mathrm{p} . u$ with the duration time of $0.2 \mathrm{~s}$. It was observed that variation of the oscillation frequencies depends on the harshness of the disturbance, the load condition and generating plant operation condition. During the generator one voltage step increase disturbance several oscillation modes exist.

Fig. 12 shows the measured signals and estimated signals for this case. Table 5 shows the damping parameters of the tie line and three dominant modes exist. It can be concluded that the tie line operation is limited by both local and inter-area oscillations. According to Lyapunov stability criterion, this system is:

- Local area 1 mode stable, since all signals in this mode have $\sigma_{i}<0$

- Local area 2 mode unstable, since all signals in this mode have $\sigma_{i}>0$

- Inter-area mode unstable, since all signals in this mode have $\sigma_{i}>0$

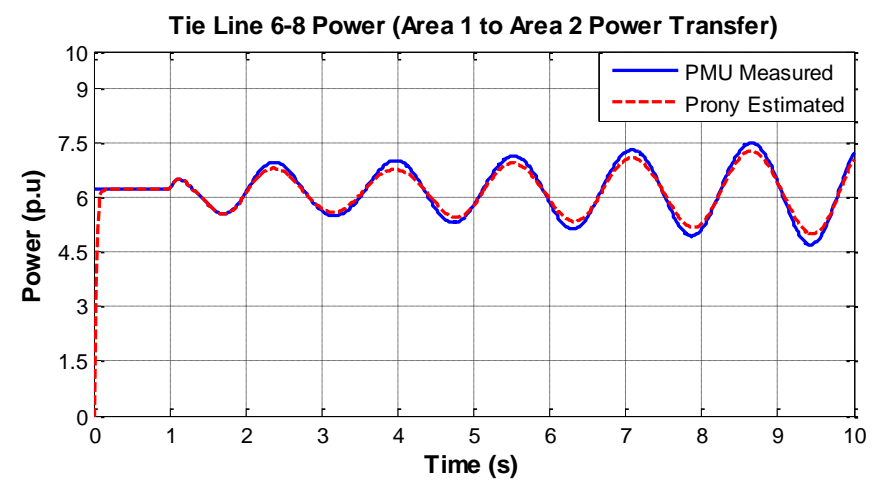

Fig. 12. Measured tie line power and estimated signal.

Table 5: Parameters of the active power flow $\left(P_{68}\right)$

\begin{tabular}{cccc}
\hline Modes & Frequency & Damping Factor & Damping Ratio \\
\hline Inter Area Mode & 0.6389 & 0.1151 & -0.02866 \\
Local Mode 1 & 1.1770 & -6.8724 & 0.68073 \\
Local Mode 2 & 1.2608 & 0.0455 & -0.00574 \\
\hline
\end{tabular}

\section{Conclusions}

This paper explored the prospect of synchronizing PMUs by means of PTP with software-only timestamp mechanism. Several tests i.e. TVE and phase error tests have been performed to verify the performances of the proposed synchrophasor solutions. The results have given clear indications on the possible use of PTP-based systems (software-only time-stamping) for the synchronization of the PMUs on the electromechanical oscillation monitoring application. Since electromechanical oscillations are relatively slow, the results suggest that the synchronization error brought by the uncertainty of the PTP software only would have minimal impact on the electromechanical oscillation monitoring application.

\section{References}

[1] Phadke A.G, Thorp J.S., Synchronized Phasor Measurements and Their Applications: Springer Science + Business Media, 2008.

[2] Shewale R, Kethineni B, Balaraju U, Bhil S, More B. Optimal placement of phasor measurement units for power system observabilty by heauristic search metheod. International Journal of Advanced Technology \& Engineering Research, 2012;2(2):128-133. 
[3] Correl K., Barendt N, Branicky M. Design considerations for software only implementations of the IEEE 1588 precision time protocol. presented at: Conference on IEEE 1588. 2005 Zurich, Switzerland.

[4] Kovacshazy T, Ferencz B. Performance Evaluation of PTPd, a IEEE 1588 implementation, on the x86 Linux platform for Typical Application Scenarios. presented at: 2012 the Instrumentation and Measurement Technology Conference (I2MTC), 2012 IEEE International Graz 2012.

[5] Lixia M, Benigni A, AFlammini A, Muscas C, Ponci F, Monti A. A Software-only PTP Synchronization for Power System State Estimation with PMUs. In: Proc. of Instrumentation and Measurement Technology Conference (I2MTC), Binjiang 2011: 1 - 6 .

[6] F. Nuqui R. State Estimation and Voltage Security Monitoring Using Synchronized Phasor Measurements. PhD dissertation. Department of Electrical Engineering. Virginia Polytechnic Institute and State University, Blacksburg, Virginia; 2001.

[7] Ahmed W. Clock Synchronization: Combining IEEE 1588 and Adaptive Oscillator Correction Method. Ms thesis. Department of Systems and Computer Engineering. Ottawa-Carleton Institute for Electrical and Computer Engineering (OCIECE): 2012.

[8] IEEE Standard for Synchrophasors for Power Systems. IEEE Standard C37.118.1-2011 (2011).

[9] Mohammadi B, Rabiei A, Moradi-Dalvand M, Mobayen S. Online inter-area oscillation monitoring in power systems using PMU data and Prony analysis. International Journal of the Physical Sciences, 2008; 6(22):5267-5272.

[10] Kundur P.S. Power Systems Stability and Control. Sydney Tokyo Toronto: McGraw-Hill, lnc; 1996. 